\title{
Episodes of strain experienced
} in the operating room: impact of the type of surgery, the profession and the phase of the operation

\author{
Sandra Keller ${ }^{1^{*}}$ (D), Steven Yule 2,3,4,5 , Douglas S. Smink ${ }^{2,4}$, Vivian Zagarese ${ }^{6}$, Shawn Safford ${ }^{7,8}$
} and Sarah Henrickson Parker ${ }^{1,6,8}$

\begin{abstract}
Background: Strain episodes, defined as phases of higher workload, stress or negative emotions, occur everyday in the operating room (OR). Accurate knowledge of when strain is most intense for the different OR team members is imperative for developing appropriate interventions. The primary goal of the study was to investigate temporal patterns of strain across surgical phases for different professionals working in the OR, for different types of operations.

Methods: We developed a guided recall method to assess the experience of strain from the perspective of operating room (OR) team members. The guided recall was completed by surgeons, residents, anesthesiologists, circulating nurses and scrub technicians immediately after 113 operations, performed in 5 departments of one hospital in North America. We also conducted interviews with 16 surgeons on strain moments during their specific operation types. Strain experiences were related to surgical phases and compared across different operation types separately for each profession in the OR.

Results: We analyzed 693 guided recalls. General linear modeling (GLM) showed that strain varied across the phases of the operations (defined as before incision, first third, middle third and last third) [quadratic $(F=47.85, p<0.001$ ) and cubic $(F=8.94, p=0.003)$ effects]. Phases of operations varied across professional groups [linear $(F=4.14, p=0.001)$ and quadratic ( $F=14.28, p<0.001)$ effects] and surgery types [only cubic effects $(F=4.92, p=0.001)$ ]. Overall strain was similar across surgery types $(F=1.27, p=0.28)$. Surgeons reported generally more strain episodes during the first and second third of the operations; except in vascular operations, where no phase was associated with significantly higher strain levels, and emergency/trauma surgery, where strain episodes occurred primarily during the first third of the operation. Other professional groups showed different strain time patterns.

Conclusions: Members of the OR teams experience strain differently across the phases of an operation. Thus, phases with high concentration requirements may highly vary across OR team members and no single phase of an operation can be defined as a "sterile cockpit" phase for all team members.
\end{abstract}

Keywords: Operating room, Strain, Stress, Tension, Phase, Surgeon, Anesthetist, Scrub technician, Circulating nurse, Student

*Correspondence: sandra.keller@insel.ch

${ }^{1}$ Fralin Biomedical Research Institute at Virginia Tech Carilion, Roanoke, VA, USA

Full list of author information is available at the end of the article

\section{Background}

OR team members of all professions experience strain as a consequence of higher workload or stressors related to the task, team or environment. Strain can 
ensue from workload (defined as external demands), demands on the working memory or mental effort during task execution [1], or from social stressors and interpersonal tensions [2-4]. The term strain describes experienced stress [5] and the response to stressors [6]. For OR team members, strain can emerge from multiple sources, including task, collaboration, technology, ambiguity and patient care related time pressure [7, 8]. It is crucial to identify phases of high strain in the OR because strain through high workload or stress was found to decrease surgical technical performance, non technical skills and to impair anesthesiologists' perceptions of relevant information [9-12]

Because strain is influenced by tasks and interactions in OR teams, strain is not constant during surgery. Phases of high strain may be followed by routine, low strain phases. Some of the more straining phases may be prospectively known, but others depend on the actual situation and events happening during the operation [13]. Identifying when phases of high workload occur during operations was a crucial step in the definition of phases of the operations during which interruptions and distractions should be minimized. Such phases are often described as sterile cockpit phases, or no interruption zones and were imported from aviation to the medical domain [14]. However, a major barrier to such intervention is that workload phases differ for different members of the OR team. In a smaller scale cardiac surgery study, anesthesia providers and circulating nurses reported high workload before, at the begin and towards the end of the surgery, whereas surgeons, and to a lesser extent scrub technicians, were most stressed during the middle, surgical repair phase of cardiac surgeries [15]. These studies suggest that phases of high strain may not be the same and do not have the same intensity for the different members of the surgical team. Because phases of high strain are closely coupled to task demands, they may also be different for different surgical procedures.

To our knowledge, besides of the study of Wadhera and colleagues [15], which was based on a small sample and a single surgical type, a systematic study to map intensity of strain across operative phases for all OR team members has not yet been undertaken. Whereas most research is based on the assumption that strain phases occur simultaneously for all team members, we know little about the temporal patterns of strain episodes for different OR team members. The aim of the present study was to identify phases of high strain for different professional groups working in the operating room (surgeons, surgery residents, anesthesiologists, scrub technicians, circulating nurses and medical students) and to compare those phases of high strain for different types of surgeries.

\section{Methods}

\section{Study design and setting}

The data were collected as part of a broader study on tense experiences in the OR. We conducted a prospective, observational, guided recall study, collecting OR team members' experiences of strain immediately after surgical procedures at one 700-bed rural hospital in North America. Data were collected over a six-month period from 2018 to 2019. We also conducted explorative interviews with surgeons. The study was approved by the Institutional Review Board of Carilion Clinic (\#2524). Prior to data collection, all participants were informed about the study and their right to refuse to participate via e-mail; verbal informed consent was obtained from all participants at data collection.

\section{Sample}

The sample consisted of 113 operations performed in five surgical departments (pediatric, general, vascular and trauma/emergency general surgery as well as gynecology). Twenty-four different surgeons led these operations. Sixteen of these surgeons (three to four surgeons from each department) were interviewed.

The typical team composition during operations included a surgeon, a surgical resident, a scrub technician, a circulating nurse, an anesthesiologist or CRNA (certified registered nurse anesthetist) and for some operations a medical student. All teams were informed about the general project and type of data collection prior to study start. Study participants were blinded as to the aims of this study, and performed their work as normal.

All team members present were invited to participate in the guided recall immediately after the operations included in the study.

\section{Measures}

\section{Post-surgery guided recall}

Based on previous work to assess affect over time in diverse settings [16], we developed a guided recall method to assess experienced strain during the operations. The guided recall was integrated into a short paper and pencil questionnaire.

Immediately after an operation, a researcher with a background in psychology (SK) invited each team member individually to draw a line representing the strain moments they experienced during the operation. The researcher explained that strain included all tense moments experienced, independent of their source. The time frame depicted the duration of the operation, ranging from shortly before incision to the end of the operation (see Fig. 1 for an example of two strain phases reported by junior surgical team members during gynecological operations). The drawing was 


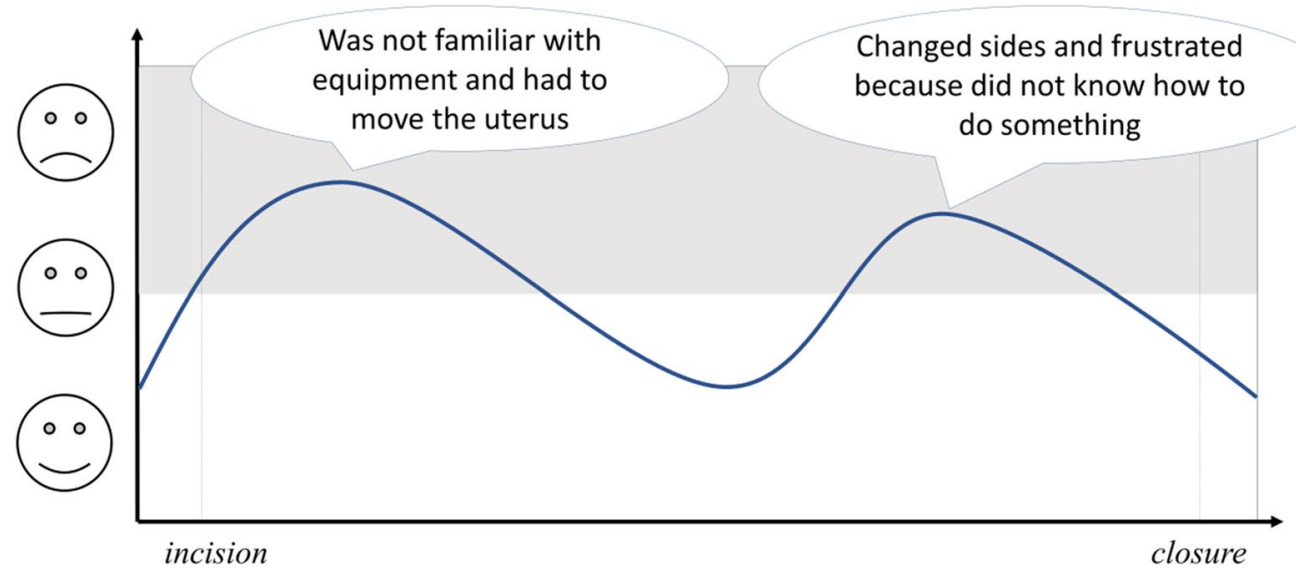

Fig. 1 Guided recall tool to measure strain during operations and examples of strain episodes

two-dimensional, with the operation timeline on the $\mathrm{x}$-axis and the strain level on the $\mathrm{y}$-axis. Smiley faces were used on the $y$-axis to represent strain, as a simple alternative to Likert scale format [17]. Team members were also invited to describe the nature of each tense moment. Responses were collected confidentially, and team members were blind to the responses of the rest of the team.

\section{Guided-recall data preparation}

We translated the drawings of each participant into numerical values, using the peak of the curve and the slope of the line towards the peak. We then defined five phases of the operations based on the drawings: a phase before incision, the first third, the second third, the last third of the intra-operative time and the phase after closure. We applied the same definition for each drawing, and the three phases between incision and closure were each calculated as one third of the distance between the incision and closure mark on the drawing (in centimeters). For each top of the curve-highest point on a curve pointing to a strain moment, independently of its height on the $y$-axis, we identified in which phase of the operation it occurred, based on the $\mathrm{x}$-axis timeline of the graph. For each team member, we calculated the total number of strain episodes per phase of the operation.

\section{Statistical analysis}

To analyze the data, we used General Linear Modeling (GLM) with repeated measures. In the initial GLM model, we first compared strain experienced by all team members (overall) across the different phases of the operations. We used GLM to analyze variation of strain levels during the different temporal phases of the operation overall, within professional groups, between professional groups, and between the different types of operations.
Our dependent variable was the number of strain episodes reported. Post-hoc tests based on least significant differences (LSD) were conducted to identify differences across different phases of the operation for each professional group. With univariate ANOVAs, we compared, for each phase of the surgery, the frequency of strain episodes across professional groups, independently of the surgery type. Significant differences across professional groups were calculated based on LSD post hoc tests.

$\mathrm{p}$ values less than 0.05 were considered statistically significant. The statistics were performed with IBM SPSS Statistics for Windows, version 25.

\section{Interviews}

The explorative interviews were conducted in a one-toone setting by one researcher (SK) and were semi-structured [18]. The interview questions related to triggers of strain episodes in the OR, and specifically when higher strain episodes were experienced during an operation. They did not refer to single operations, but to the general experiences of a participant. The interviews were audio-recorded and transcribed. We performed a content analysis to identify phases of the operation that were described as associated with higher strain levels.

\section{Results}

\section{Descriptive results}

We collected a total of 693 guided recalls from OR team members after the 113 operations included in the study, with a mean number of guided recalls per operation of 6 . The overall mean duration of the operations was $78 \mathrm{~min}$ $(\mathrm{SD}=62 \mathrm{~min}$; Table 1). The number of participants for each profession and surgery type varied with response rates ranging from 93.8 to $97.3 \%$ (Table 2). The most frequent reason for missing responses was that a team 
Table 1 Number of operations included for each surgery type and mean duration

\begin{tabular}{lccl}
\hline Surgery type & $\mathbf{n}$ & Mean duration & SD duration \\
\hline Pediatric & 23 & 49.09 & 40.34 \\
Gynecology & 23 & 109.43 & 92.31 \\
General surgery & 22 & 82.64 & 59.98 \\
Trauma/emergency & 23 & 82.30 & 44.19 \\
Vascular & 22 & 68.14 & 44.03 \\
Total & 113 & 78.37 & 61.78 \\
\hline
\end{tabular}

member could not be asked to fill out the guided recall (e.g. because he/she left the OR early). Three individuals opted to not complete the guided recall.

\section{Strain events across different phases of the surgery}

The 693 guided recalls collected contained a total of 452 strain events. Examples of strain episodes reported included clinical, interpersonal, and systemic aspects (e.g. difficult dissection of a hernia sac, too busy to make sure that everything is correct, unsure what to do or noise in the hallway during induction). No strain episode was reported for the phase of the operation immediately after wound closure. For the rest of this analysis, we excluded this phase of the operation and included only four phases: the time before incision (phase 1), the first third of the operation (phase 2), the second third of the operation (phase 3) and the last third of the operation (phase 4). Frequency, means and standard deviation of strain events for each phase of the operation are displayed in Table 3. The frequency of strain varied significantly across the four phases of the operation $(\mathrm{F}=52.75$, $\mathrm{p}<0.001 ; \mathrm{F}=7.29, \mathrm{p}=0.007$, respectively quadratic and cubic effects). Strain was most frequently reported in the middle third of the operation (phase 3), followed by the first third of the operation (Phase 2) (see Additional file 1 for the detail of the post hoc tests).
Table 3 Overall strain episodes reported in the different phases of the operations

\begin{tabular}{lrllll}
\hline & $\mathbf{n}$ & Minimum & Maximum & Mean & SD \\
\hline Before incision & 89 & 0.00 & 5.00 & 0.79 & 1.03 \\
First third & 138 & 0.00 & 7.00 & 1.22 & 1.42 \\
Second third & 168 & 0.00 & 8.00 & 1.49 & 1.64 \\
Last third & 57 & 0.00 & 3.00 & 0.50 & 0.71 \\
Total & 452 & & & & \\
\hline
\end{tabular}

$\mathrm{N}=113$ operations

In a GLM model, we tested the effects of the phase of the operation, the professional group and the type of operation on experienced strain episodes. Results of within subjects models revealed a significant effect of the phase of the operation on the frequency of strain episodes [quadratic $(\mathrm{F}=47.85, \mathrm{p}<0.001)$ and cubic $(\mathrm{F}=8.94$, $\mathrm{p}=0.003$ ) effects] (Additional file 2). These effects varied depending on the professional group [linear $(\mathrm{F}=4.14$, $\mathrm{p}=0.001)$ and quadratic $(\mathrm{F}=14.28, \mathrm{p}<0.001)$ effects]. This indicates that the different professional groups did not experience strain episodes during the same phases of the operation. The strain time patterns of the professional groups also varied depending on the surgery type $(\mathrm{F}=4.92, \mathrm{p}=0.001$, only cubic effects), suggesting that different surgical types show a different temporal pattern of strain. However, overall strain during different types of operations was similar $(\mathrm{F}=1.27, \mathrm{p}=0.280$, see Additional file 3 for between subject effects).

Mean strain episodes per phase of the operations and significant differences are presented in Table 4 and illustrated in Fig. 2; the complete post hoc tests are presented in Additional file 4. We present some examples based on the statistically significant differences revealed by posthoc tests based on LSD (least significant differences).

Surgeons reported generally more strain episodes during the first and second third of the operation (phases 2 and 3). Exceptions were vascular surgery, where no phase

Table 2 Number of study participants for each profession per surgery type

\begin{tabular}{|c|c|c|c|c|c|c|c|}
\hline & Surgeons & Residents & $\begin{array}{l}\text { Medical } \\
\text { students }\end{array}$ & $\begin{array}{l}\text { Scrub } \\
\text { technicians }\end{array}$ & $\begin{array}{l}\text { Circulating } \\
\text { nurses }\end{array}$ & $\begin{array}{l}\text { Anesthesiology } \\
\text { specialists }\end{array}$ & Total \\
\hline Pediatric & 24 & 22 & 14 & 25 & 26 & 29 & 140 \\
\hline Gynecology & 23 & 29 & 15 & 25 & 33 & 20 & 145 \\
\hline General surgery & 22 & 20 & 10 & 37 & 27 & 27 & 143 \\
\hline Trauma/emergency & 23 & 37 & 14 & 23 & 31 & 29 & 157 \\
\hline Vascular & 23 & 12 & 0 & 26 & 26 & 21 & 108 \\
\hline Total $^{\mathrm{a}}$ & 115 & 120 & 53 & 136 & 143 & 126 & 693 \\
\hline
\end{tabular}

a Some participants filled out a guided recall for several operations. Also, when more than one team member of a same professional group was present during an operation, all were invited to fill out a guided recall. This explains that the total number of participants exceeds the number of operations included, except for the medical students 


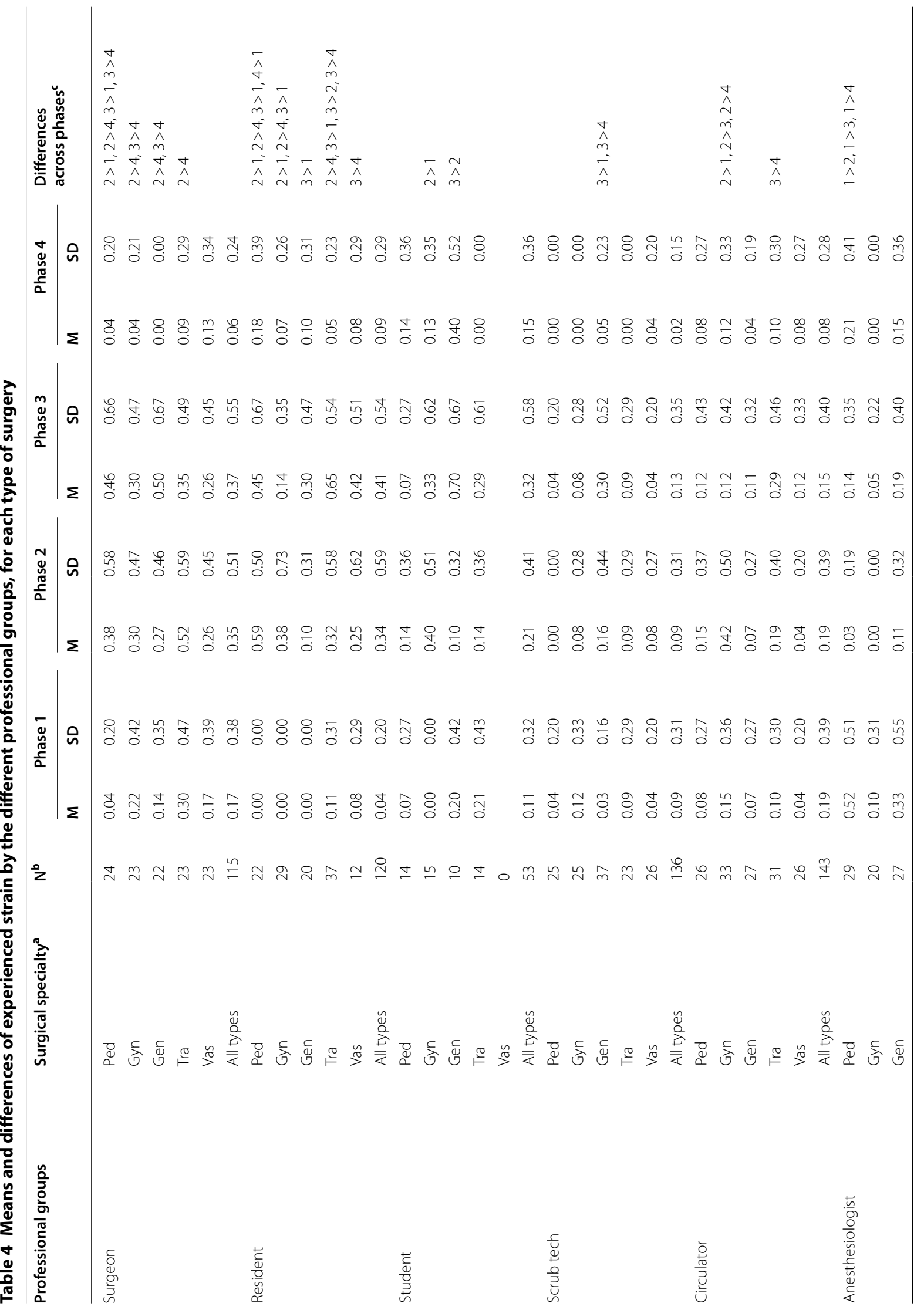




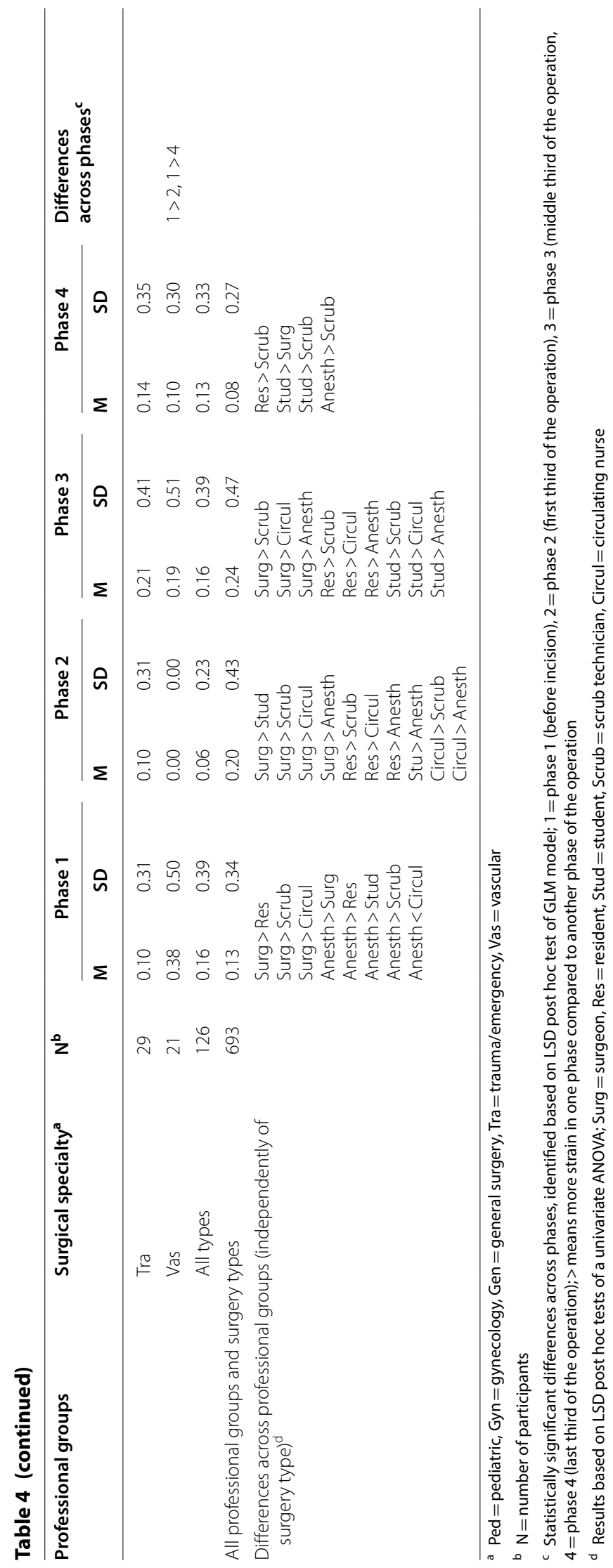




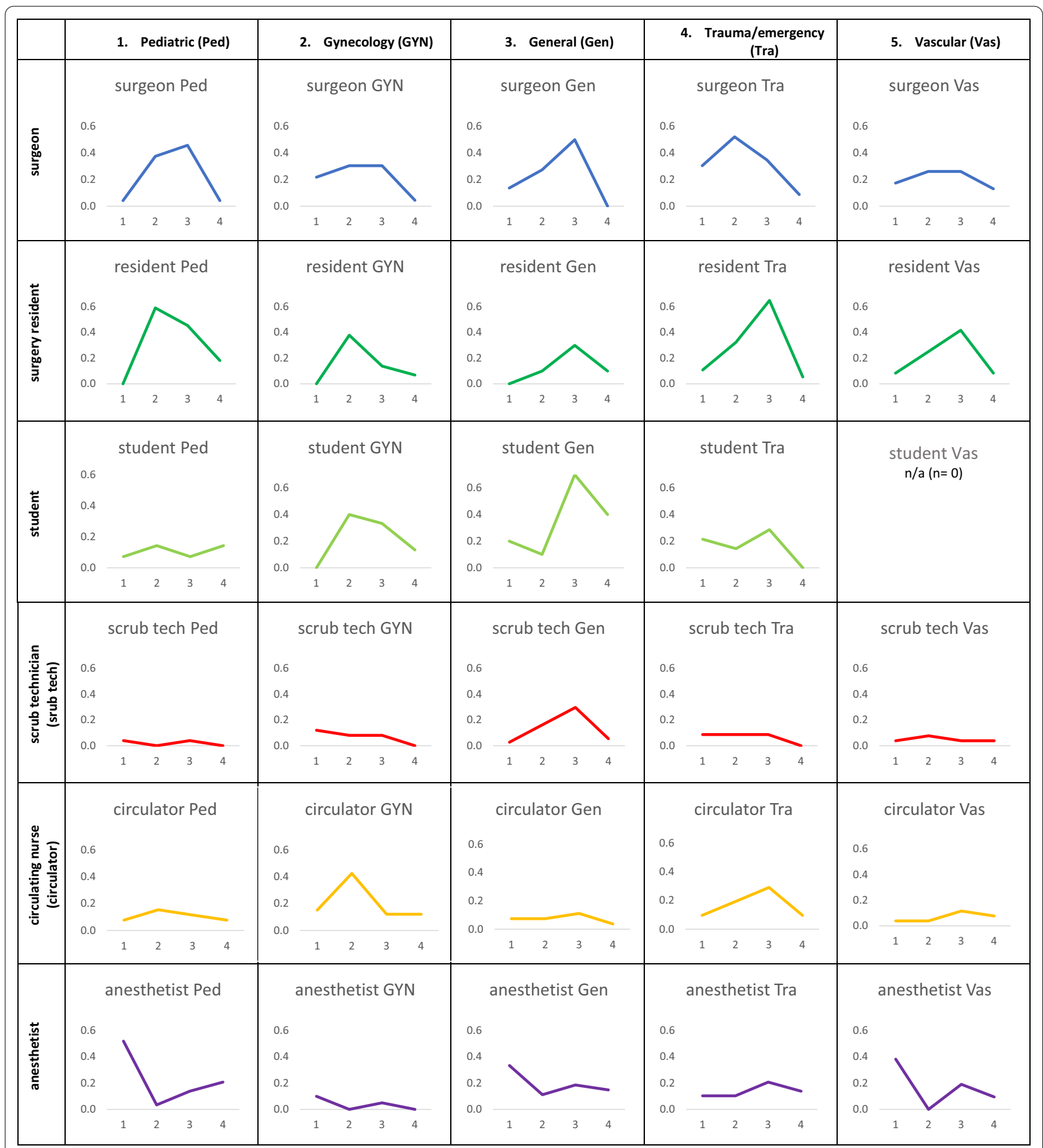

Fig. 2 Mean frequency of strain events reported during the four phases of the operations. Note: $x$-axis: 1 = Phase 1, before incision, $2=$ Phase 2 , begin (first third of the operation), $3=$ Phase 3, middle (middle third of the operation), $4=$ Phase 4 , end (last third of the operation); $y$-axis: scale representing mean frequency of strain episodes reported by the participants

was associated with significantly higher strain levels, and trauma/emergency surgery, where strain episodes where concentrated during the first third of the operation. Residents showed similar patterns. However, residents experienced mostly strain episodes during the middle part of the operation (phase 3), in particular in trauma/ emergency surgery. Interestingly, scrub technicians experienced more frequent strain episodes in the middle part 
of general surgeries (phase 3), whereas circulating nurses experienced more strain in the first third of gynecology operations (phase 2) and middle third of trauma/emergency operations (phase 3). Anesthetists reported more strain episodes only in the first phase of paediatric and vascular operations, i.e. during induction. Of note, there was a non-significant trend towards more strain during the last phase of pediatric operations $(p=0.057)$ (see Additional file 4) but not for other surgery types.

Differences across professional groups separately for each phase, independent of surgical type (see bottom part of Table 4), showed that anesthesiologists experienced more strain episodes in the phase before incision (i.e. induction) than most other professional groups. Surgeons, residents and students were more likely to experience strain episodes in phase 2 and 3. Medical students and residents experienced higher strain than other professional groups towards the end of the operation (see Additional file 5 for the detail of the post hoc tests).

\section{Results from the qualitative interviews}

The qualitative interviews revealed that the surgeons could identify phases of the operation associated with higher strain levels. A synthesis of the results (Fig. 3) showed that surgeons from different surgical specialties identified different phases of their operations as related to an expected increase in strain.

The phases immediately before the operation and the start of the operation were mentioned as associated with high strain by the gynecology and the trauma/emergency surgeons. The reason for more strain was the particularly important preparation and organization of the material before starting the surgery. The first phase was described

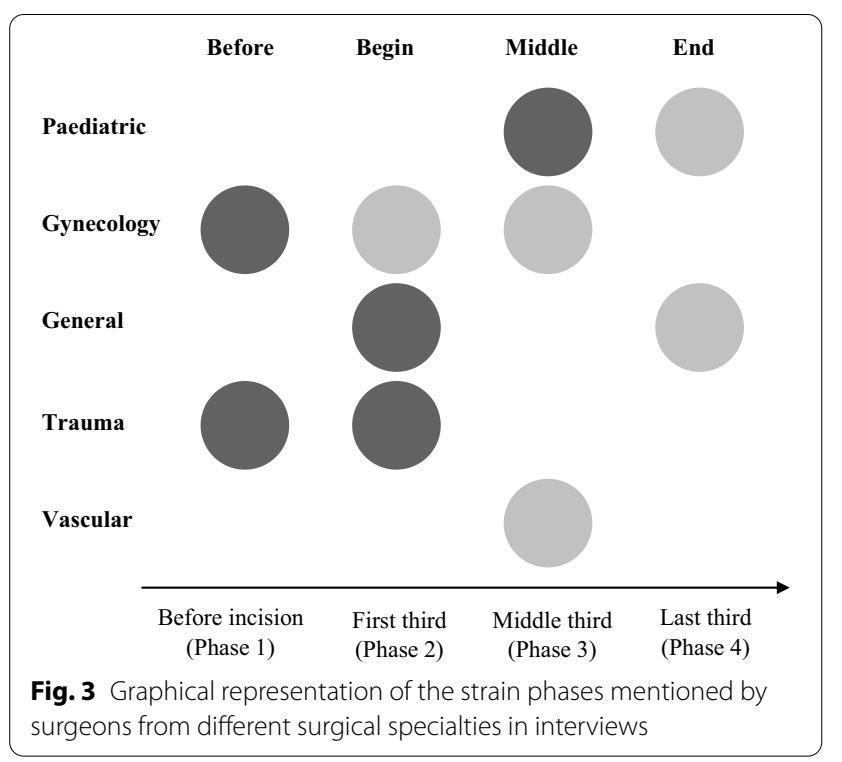

as particularly straining again by the trauma/emergency surgeons, gynecology and, to a somewhat lesser extent, by general surgeons. Trauma surgeons mentioned the uncertainty and decision making as an important challenge in the first phase, because detailed planning of the surgery was not possible beforehand. A general surgeon also mentioned that getting everyone's attention for the safety checks at the start of the operation as a critical part of the process. The middle phase of the operation was mentioned to be associated with higher strain levels only by the pediatric, gynecology and vascular surgeons mainly due to higher technical demands. The end of the operation was not mentioned as a high strain phase with the exception of pediatrics and general surgeons; reasons for strain in this phase were an increased level of distractions and organizational challenges associated with patient transfer to the post operative care unit.

\section{Discussion}

Our results showed that strain episodes experienced by OR team members varied as a function of the phase of the operation, professional group, and of the surgical specialty. Overall, the guided recall and the interview results converged well. Thus, professional groups in the OR experience high strain in different phases of the operation even within the same surgical type. In addition, phases of strain vary across surgical types. We can thus refute a general phase model of strain for all surgeries or across different professions.

\section{Different professional groups experience different strain phases}

Consistent with previous research [19], in this study, surgeons showed overall more strain than all other team members during most phases of the operation. Across all surgeries, surgeons reported higher strain levels during the middle part of the surgeries, as shown in previous studies $[15,20]$. However, when types of surgeries were considered, the pattern was more complex. Trauma surgeons reported higher strain levels at the beginning, mentioning organization of resources, uncertainty about the operation and decisions to be made, in line with previous research that showed that novel situations are likely to trigger uncertainty in surgeons [21]. For most of the surgical specialties, the guided recall and interview data coincide. However, the general surgeons' guided recalls showed a clear peak of strain during the middle third of the surgeries, whereas in the interview, the surgeons labeled the first and last third of the surgery as straining. Interestingly, the reasons given in the interviews included organizational constraints, teamwork and distractions as predictors of strain. 
Residents' reports of more frequent strain phases only partly mirrored the surgeons' strain patterns. For most operations, residents reported more strain in the middle third of the operation. Again, this coincides with the phase of highest task demands, because in the middle third, residents often operate actively under the supervision of the surgeon. This was particularly the case in trauma/emergency surgery. Similarly, medical students reported more strain during the phases in which they were more actively involved in the operation and collaborating closer with the team (e.g. during the last phase of the operation, when they help closing the wound).

For most operations, our results showed less frequent strain for the nurses (scrub technicians and circulators). Previous research had identified the phase before the start of the surgery (e.g. material preparation) [22] and the end of the operation [15] as particularly straining for nurses, because the phases of preparation and the final counts are critical tasks. Our results where more differentiated and again revealed different phase patterns for different procedures: During general surgery procedures, scrub technicians showed higher levels of strain in the middle phase, mirroring the pattern of the surgeons and potentially due to the particularly high number of instruments in general surgery.

Circulating nurses showed higher amount of strain in the first third of the operation in gynecology operations, probably because of the complex organization of the material, mentioned by the surgeons in the interviews and found to be a major source of interruptions, occurring mostly in the first thirty minutes of gynecology operations [23]. In trauma/emergency surgeries, they reported more strain in the middle third, potentially because they had to prepare new technical equipment after the operative decisions were made by the surgeons.

\section{Anesthetists}

Most previous studies found the induction and emergence phases related to strain for the anesthetists and parallels were made with take-off and landing in aviation $[15,24,25]$. Our results were more differentiated. We found that only induction (the first phase) but not emergence, was associated with more strain. Pediatric surgery procedures were an exception; the last phase of the surgery, emergence from anesthesia, was associated with more strain, albeit only as a statistical trend. Also, strain levels at induction depended on the type of surgery, with higher strain at induction particularly for pediatric and vascular surgery patients. An explanation could be that those patients require particularly complex work processes.

Overall, this study showed that often made assumptions do not hold: The different members of the surgical team did not experience high strain at the same time during the same procedure. In addition, there is no overall temporal pattern of strain even within a profession, because the surgical type heavily influences strain patterns.

\section{Guided reports to measure strain}

The data were collected immediately after surgeries. This enhances the external validity of the study, as previous studies on this topic were often based on simulations, did not compare different professional groups [1], or were based on general questionnaires [15]. The methodology allowed to capture strain episodes emanating from different sources (cognitive, emotional, teamwork-related). Specific questionnaires often concentrate on one source of strain. For example, the NASA-TLX or SURG-TLX tool specifically measure cognitive workload [1]. Collecting the data immediately after the operation helped minimize recall and memory biases often associated with self-report [26]; it also allowed to capture strain related to stress and negative emotions experienced by OR teams and detrimental for surgical performance [11]. The results from the explorative interviews mostly coincided with the results of the guided recalls and offered additional information to interpret the data.

Our study also has limitations. The operations included were performed in a single center and operations performed within a same department were relatively heterogenous. A replication and finer distinction of surgical type in several centers would allow to show more diverse patterns, but also to disentangle strain related to the task and influencing factors associated with the organization, resources, and staffing. Further, we relied solely on reports of the participants. New technological developments allow to measure strain in terms of physiological changes over time, in particular in relation to the stress experienced by the OR teams (e.g. heart rate variability) $[1,9]$. Although, these new technologies are not likely to replace data collected based on subjective experiences of OR team members, they may measure complementary facets of strain [27].

\section{Conclusions}

Our results support the claim of previous researchers to better take into account task requirements of different professional groups $[15,28,29]$ and the types of surgical procedure [12] when analyzing strain of surgical team members. A better awareness of these complex patterns may also inform interventions to reduce distractions and interruptions, particularly during critical moments [25], during which workload should not unnecessarily be added [30]. 


\section{Supplementary information}

Supplementary information accompanies this paper at https://doi. org/10.1186/s12893-020-00937-y.

Additional file 1. Post hoc test showing differences across phases of the operations over all professions and all operation types.

Additional file 2. Results of the GLM Model for within subjects effects. Additional file 3. Results of the GLM Model for between subjects model. Additional file 4. Post hoc tests from the GLM model comparing differences across phases of the surgery for different professions and operation types.

Additional file 5. Post-hoc tests from Univariate Anovas comparing frequency of strain reported by the different professions, across all types of operations.

\section{Abbreviations}

OR: Operating room; GLM: General linear modeling; ANOVA: Analysis of variance.

\section{Acknowledgements}

We thank Whitney DeLong for her support in the study coordination, Patty Parker for her advice and help in organizing data collection, the head anesthesiologists and head nurses at the institution where the study took place and all our study participants. We thank Franziska Tschan and Guido Beldi for reviewing and commenting an earlier version of this manuscript.

\section{Authors' contributions}

SK, SHP, SY and DSS were involved in the conception and design of the study. SK, SS and VZ participated in the data acquisition process. SK drafted the first version of the manuscript, that was critically revised by all authors. All authors read and approved the final manuscript.

\section{Funding}

This work was sponsored by a grant of the Swiss National Science Foundation (SNSF), Grant Number P2NEP1 178574. The funding body had no role in the design of the study and collection, analysis, and interpretation of data and in writing the manuscript.

\section{Availability of data and materials}

The datasets generated and analyzed during the current study are not publicly available to protect the confidentiality of the participants but are available from the corresponding author on reasonable request and approval of the ethics committee of Carilion Clinic

\section{Ethics approval and consent to participate}

The ethics committee of Carilion Clinic approved the study (study \#2524). Verbal informed consent was obtained from all the participants because the data collected were de-identified. Prior to data collection, participants were informed about the study and of their right to refuse to participate in the study via e-mail. This procedure was approved by the ethics committee.

\section{Consent for publication}

Not applicable.

\section{Competing interests}

The authors declare that they have no competing interests.

\section{Author details}

${ }^{1}$ Fralin Biomedical Research Institute at Virginia Tech Carilion, Roanoke, VA, USA. ${ }^{2}$ Center for Surgery and Public Health, Brigham and Women's Hospital, Boston, MA, USA. ${ }^{3}$ STRATUS Center for Medical Simulation, Boston, MA, USA. ${ }^{4}$ Department of Surgery, Brigham and Women's Hospital, Harvard Medical School, Boston, MA, USA. ${ }^{5}$ Department of Clinical Surgery, University of Edinburgh, Edinburgh, Scotland, UK. ${ }^{6}$ Department of Psychology, Virginia Tech, Blacksburg, VA, USA. ${ }^{7}$ Division of Paediatric Surgery, Virginia Tech Carilion School of Medicine, Roanoke, VA, USA. ${ }^{8}$ Center for Simulation, Research and Patient Safety, Carilion Clinic, Roanoke, VA, USA.
Received: 29 July 2020 Accepted: 29 October 2020

Published online: 07 December 2020

\section{References}

1. Dias RD, Ngo-Howard MC, Boskovski MT, Zenati MA, Yule SJ. Systematic review of measurement tools to assess surgeons' intraoperative cognitive workload. Br J Surg. 2018;105(5):491-501.

2. Lingard L, Reznick R, Espin S, Regehr G, DeVito I. Team communications in the operating room: talk patterns, sites of tension, and implications for novices. Acad Med. 2002;77(3):232-7.

3. Keller S, Tschan F, Semmer NK, Timm-Holzer E, Zimmermann J, Candinas D, Demartines N, Hübner M, Beldi G. "Disruptive behavior" in the operating room: a prospective observational study of triggers and effects of tense communication episodes in surgical teams. PLoS ONE. 2019;14(12):e0226437.

4. Booij LH. Conflicts in the operating theatre. Curr Opin Anesthesiol. 2007;20(2):152-6.

5. Carayon P, Zijlstra F. Relationship between job control, work pressure and strain: Studies in the USA and in The Netherlands. Work \& Stress. 1999;13(1):32-48.

6. Sonnentag S, Frese M. Stress in organizations. In: Handbook of psychology. 2003. p. 453-91.

7. Poolton JM, Wilson MR, Malhotra N, Ngo K, Masters RS. A comparison of evaluation, time pressure, and multitasking as stressors of psychomotor operative performance. Surgery. 2011;149(6):776-82.

8. $\mathrm{Ng} \mathrm{R}$, Chahine S, Lanting B, Howard J. Unpacking the literature on stress and resiliency: a narrative review focused on learners in the operating room. J Surg Educ. 2019;76(2):343-53.

9. Zenati MA, Leissner KB, Zorca S, Kennedy-Metz L, Yule SJ, Dias RD. First reported use of team cognitive workload for root cause analysis in cardiac surgery. In: Seminars in thoracic and cardiovascular surgery. Elsevier; 2019. p. 394-96.

10. Jones C, Fawker-Corbett J, Groom P, Morton B, Lister C, Mercer S. Human factors in preventing complications in anaesthesia: a systematic review. Anaesthesia. 2018;73:12-24.

11. Arora S, Sevdalis N, Nestel D, Woloshynowych M, Darzi A, Kneebone R. The impact of stress on surgical performance: a systematic review of the literature. Surgery. 2010;147(3):318-330.e316.

12. Weigl M, Stefan P, Abhari K, Wucherer P, Fallavollita P, Lazarovici M, Weidert S, Euler E, Catchpole K. Intra-operative disruptions, surgeon's mental workload, and technical performance in a full-scale simulated procedure. Surg Endosc. 2016;30(2):559-66.

13. Moulton C-A, Regehr G, Lingard L, Merritt C, MacRae H. 'Slowing down when you should': initiators and influences of the transition from the routine to the effortful. J Gastrointest Surg. 2010;14(6):1019-26.

14. Filer HM, Beringuel BL, Frato KM, Anthony MK, Saenyakul P. Interruptions in preanesthesia nursing workflow: a pilot study of pediatric patient safety. J PeriAnesth Nurs. 2017;32(2):112-20.

15. Wadhera RK, Parker SH, Burkhart HM, Greason KL, Neal JR, Levenick KM, Wiegmann DA, Sundt TM III. Is the "sterile cockpit" concept applicable to cardiovascular surgery critical intervals or critical events? The impact of protocol-driven communication during cardiopulmonary bypass. J Thorac Cardiovasc Surg. 2010;139(2):312-9.

16. Kuppens P, Van Mechelen I, Nezlek JB, Dossche D, Timmermans T. Individual differences in core affect variability and their relationship to personality and psychological adjustment. Emotion. 2007;7(2):262-74.

17. Toepoel V, Vermeeren B, Metin B. Smileys, stars, hearts, buttons, tiles or grids: influence of response format on substantive response, questionnaire experience and response time. Bull Sociol Methodol. 2019;142(1):57-74.

18. Adams E. The joys and challenges of semi-structured interviewing. Community Pract. 2010;83(7):18-22.

19. Wheelock A, Suliman A, Wharton R, Babu E, Hull L, Vincent C, Sevdalis N, Arora $\mathrm{S}$. The impact of operating room distractions on stress, workload, and teamwork. Ann Surg. 2015;261(6):1079-84.

20. Katz P. Ritual in the operating room. Ethnology. 1981;20:335-50. 
21. Cristancho SM, Apramian MT, Vanstone M, Lingard L, Ott M, Novick RJ. Understanding clinical uncertainty: what is going on when experienced surgeons are not sure what to do? Acad Med. 2013;88(10):1516.

22. Clark GJ. Strategies for preventing distractions and interruptions in the OR. AORN J. 2013;97(6):702-7.

23. Yoong W, Khin A, Ramlal N, Loabile B, Forman S. Interruptions and distractions in the gynaecological operating theatre: irritating or dangerous? Ergonomics. 2015;58(8):1314-9.

24. Broom MA, Capek AL, Carachi P, Akeroyd MA, Hilditch G. Critical phase distractions in anaesthesia and the sterile cockpit concept. Anaesthesia. 2011;66(3):175-9.

25. Campbell G, Arfanis K, Smith AF. Distraction and interruption in anaesthetic practice. Br J Anaesth. 2012;109(5):707-15.

26. Raphael K. Recall bias: a proposal for assessment and control. Int J Epidemiol. 1987;16(2):167-70.

27. Kennedy-Metz LR, Dias RD, Stevens RH, Yule SJ, Zenati MA. Analysis of mirrored psychophysiological change of cardiac surgery team members during open surgery. J Surg Educ (in press).
28. Jothiraj H, Howland-Harris J, Evley R, Moppett I. Distractions and the anaesthetist: a qualitative study of context and direction of distraction. $\mathrm{Br}$ J Anaesth. 2013;111(3):477-82.

29. Wahr JA, Prager RL, Abernathy J, Martinez EA, Salas E, Seifert PC, Groom RC, Spiess BD, Searles BE, Sundt TM. Patient safety in the cardiac operating room: human factors and teamwork a scientific statement from the American Heart Association. Circulation. 2013;128(10):1139-69.

30. Weigl M, Weber J, Hallett E, Pfandler M, Schlenker B, Becker A, Catchpole K. Associations of intraoperative flow disruptions and operating room teamwork during robotic-assisted radical prostatectomy. Urology. 2018;114:105-13.

\section{Publisher's Note}

Springer Nature remains neutral with regard to jurisdictional claims in published maps and institutional affiliations.
Ready to submit your research? Choose BMC and benefit from:

- fast, convenient online submission

- thorough peer review by experienced researchers in your field

- rapid publication on acceptance

- support for research data, including large and complex data types

- gold Open Access which fosters wider collaboration and increased citations

- maximum visibility for your research: over $100 \mathrm{M}$ website views per year

At BMC, research is always in progress.

Learn more biomedcentral.com/submissions 